\section{An Infrared Microspectroscopist Speaks}

Howard J. Humecki

Senior Research Chemist, McCrone Associates, Inc.

They're smart, these light microscopists! And sneaky too! They didn't bother me when I struggled trying to squeeze a little more information from a tiny specimen. They were the purists who scoffed at my attempts to identify little bits of organic stuff by infrared spectroscopy. They only needed their polarized light microscopes (PLM) and a few reagents to conduct microchemical tests. After all, they reasoned, if chemical microscopists couldn't identify something merely by looking at it, it probably wasn't worth knowing. Well, times have changed! Microanalysts in all fields are exploring the possibilities of infrared microspectroscopy to gather information that was difficult or impossible to obtain before. Foremost among those jumping on the bandwagon are the light and electron microscopists who have realized the potential of this tool now entering what might be called the third generation in a span of 15 years.

Forensic microscopists have taken to infrared microspectoscopy (not infrared microscopy) like ducks to water. Paint chip analysis by optical and $x$-ray methods is now augmented by binder analysis, by microspectroscopy. Multi-layers can be scraped and analyzed coat by coat or embedded in a polymer and cross-sectioned. Synthetic fibers can be analyzed in detail as well. Bits of evidence that may have demanded hours of study to characterize can now be identified in a matter of minutes by microspectroscopy.

Packaging people and those in the films and coatings industry have benefitted as well. They can now prepare cross sections of multilayered films and coatings and study the composition layer by layer. Programmable stages are available to follow the migration of additives from one layer to the next or to follow, for example, the degree of UV cured resins as a function of depth from the surface. Minute defects can be excised and identified and, in some cases, analyzed in situ. These marvelously precise mechanical stages are available for step-scanning through a thin section or rastering through a twodimensional cross section to produce a three-dimensional image showing the concentration of various components by their absorption at selected wavelengths. This technique has been employed in medical research to study the distribution and composition of cholesterol esters in arteries. It has even been employed in the reflectance mode to study mineral phases.

Microscopists in art conservation are using microspectroscopy to study varnish and binders in artist's paint as well as the degradation of polymers and coatings by techniques that are quite similar to those used in forensics and packaging. They could benefit by an exchange of ideas. It's a pity they rarely talk to one another.

In the pharmaceutical field; combined PLM, x-ray diffraction, and spectoscopy have been employed to follow changes in polymorphism. Countless particles have been filtered from solutions and identified by microspectroscopy. Spots of discolored material on the surface of tablets and tablet coatings can sometimes be analyzed in situ using micro ATR objectives.

Libraries are available that can search through thousands of spectra in a matter of seconds. The microscopists need only issue a few directions and the computer spits out a hit list of possible IDs. They're in fat city!

But wait! What if the sample happens to be an intractable goo consisting of so many components that no single reference spectrum will provide a close match? Pick the spectum of the closest match and subtract, then search what's left you say. Fine in theory, but those who have tried know that spectra included in libraries generally range from 100 to $<10 \%$ transmission - far from the linear range for Beers law compliance. The result is a different spectrum showing both positive and negative peaks.

This is usually the last straw for the part-time spectroscopists. They need help from someone who has muddled this way through many similar problems without the aid of computer searches. Someone who in the murky depths of his mind has the recollection of having seen similar combinations of absorption bands. Someone who can find urethane bands in a modified alkyd paint chip contaminated with pigments and miscellaneous debris. They come to someone like me, a professional with expertise in spectroscopy, just as they have expertise in optical or electron microscopy. It makes me feel good. And I like it.

\section{Calling All SEM Users ... . We'd Like to Pick Your Brains!}

Fred Schamber, RJ Lee Group, Inc.

Some months ago, I received a call from an editor associated with a prominent technical publishing company. This individual had read a SEMrelated article I had recently authored and was inquiring into the possibility of producing a SEM handbook. The idea intrigued me, as it did several others I spoke with. We agreed that there were several fine SEM texts currently available, but we felt there was still a gap between the "theory" of SEM as it is usually presented in texts, and the day-to-day practice of using a SEM to solve problems. As an exercise, we were each able to list several topics which we have found necessary to explain to other SEM users with some regularity, and for which we were unaware of any reference source. Mostly, these things fell into the category of "tricks" and tactics we had learned, pitfalls we had learned to avoid, or concepts for which we had a particular insight. This is important stuff, we all agreed, but not the sort of thing one could fill a book with ... a at least we alone couldn't. That's when we got the idea of tapping the experience of the larger SEM community.

What we would like to do is to capture a lot of the "street smarts" that currently exists in the community of SEM users and publish it in a handbook form. It is our belief that anyone who has operated a SEM for any period of time has learned things which someone else could benefit form. Even things which may be highly specific to a particular set of circumstances may give someone else insight into a different problem they are facing. We hope that a lot of the "old hands" from the SEM community will come forward with contributions which explain how they used to get work done in the "old days" before instruments and techniques got so sophisticated. Similarly, the new generation of field-emission and low vacuum instruments is creating a whole new set of questions and associated expertise

We intend for this handbook to focus on the hands-on aspects of scanning electron microscopy and related analytical and instrumental techniques. A partial list of topics of interest would include: sample selection, preparation, and presentation; tactics for approaching analytical problems of various kinds (including multi-technique approaches); $x$-ray microanalysis (EDS and WDS); imaging detectors of all kinds and how to use them effectively; instrument maintenance (including vacuum know-how); techniques for optimizing instrument performance; etc. In general, we are looking for any kind of contribution which you think warrants passing on to others: tricks which simplify your work; unusual or particularly helpful insights; useful fixtures, tools, or modifications; artifacts you have encountered; difficult samples and how you have handled them; tips on how to "tune" for the best picture; etc.

If you have suggestions - for contributions you could make, for topics you would like to see someone else cover, for specific individuals that you think should be contacted for contributions, or if you just want to express yourself about this concept, please address such communications to:

\section{Atten: SEM Handbook}

c/o RJ Lee Group, Inc.

515 Pleasant Valley Road

Trafford, PA 15085

$$
\text { or FAX: (412)744-0506 }
$$

We are looking for contributions that can range from a "pithy paragraph" to ones of chapter length. If you're busy right now, just send a couple of sentences which outline what you have in mind, but please respond soon so that we can gauge response. Please don't send any materials that you need to have returned or which would be proprietary. We do plan to acknowledge all contributors, so please include your name and address.

We can't yet be very specific about publication format, criteria for selection, ultimate size or scope of the handbook, or a lot of other practical matters. We will first see what you and others like you are willing to contribute and then see how it all fits together. With your help, we feel that this handbook can make a lasting contribution to the practice of scanning electron microscopy. 